\title{
An Xp22.12 microduplication including RPS6KA3 identified in a family with variably affected intellectual and behavioral disabilities
}

\author{
Ayumi Matsumoto ${ }^{1}$, Mari Kuwajima ${ }^{1}$, Kunio Miyake ${ }^{2}$, Karin Kojima ${ }^{1}$, Naomi Nakashima ${ }^{1}$, Eriko F Jimbo ${ }^{1}$, \\ Takeo Kubota ${ }^{2}$, Mariko Y Momoi ${ }^{1}$ and Takanori Yamagata ${ }^{1}$
}

The ribosomal protein S6 kinase, $90 \mathrm{~kb}$, polypeptide 3 gene (RPS6KA3) is responsible for Coffin-Lowry syndrome (CLS), which is characterized by intellectual disability (ID) and facial and bony abnormalities. This gene also affects nonsyndromic X-linked ID and nonsyndromic X-linked ID without bony abnormalities. Two families have been previously reported to have genetic microduplication including RPS6KA3. In the present study, we used array-comparative genomic hybridization (CGH) analysis with Agilent Human genome CGH $180 \mathrm{~K}$ and detected a 584-kb microduplication spanning 19.92-20.50 Mb of Xp22.12 (including RPS6KA3) in the members of one family, including three brothers, two sisters, and their mother. The 15-year-old male proband and one of his brothers had mild ID and localization-related epilepsy, whereas his other brother presented borderline intelligence quotient (IQ) and attention-deficit-hyperactivity disorder (ADHD). One sister presented pervasive development disorder (PDD). Analysis of this family suggests that RPS6KA3 duplication is responsible for mild ID, ADHD, and localization-related epilepsy, and possibly for PDD.

Journal of Human Genetics (2013) 58, 755-757; doi:10.1038/jhg.2013.88; published online 29 August 2013

Keywords: Coffin-Lowry syndrome; epilepsy; intellectual disability; microduplication; pervasive development disorder; RPS6KA3; Xp22.12

Coffin-Lowry syndrome (CLS), first described by Coffin in 1966, is an X-linked semidominant syndrome characterized by severe intellectual disability (ID), facial dysmorphism, and progressive skeletal deformities. ${ }^{1}$ The gene responsible for CLS is the ribosomal protein S6 kinase, $90 \mathrm{kD}$, polypeptide 3 (RPS6KA3), which also causes nonsyndromic X-linked ID. ${ }^{2}$ To date, there have been reports of two families with mild ID and Xp22.12 microduplications that include RPS6KA3. ${ }^{3,4}$ Here, we report on a $584-\mathrm{kb}$ microduplication of Xp22.12 including RPS6KA3 identified in a family. The phenotypic appearance was variable among the affected members of this family. Family members presented mild ID, attention-deficit-hyperactivity disorder (ADHD), and other phenotypes, including localizationrelated epilepsy that was not described in the previously reported families.

The proband (IV-6) is a 15-year-old male (Figure 1) with no facial dysmorphism or bony abnormality. He started walking alone at 17 months and speaking few words at 18 months of age. At 6 years of age, his intelligence quotient (IQ) was 63 according to the Binet Intelligence Scales, Fifth Edition. He had difficulty remaining seated and concentrating on task completion during class, and he sometimes suffered panic attacks. He was diagnosed with ADHD according to the Diagnostic and Statistical Manual of Mental Disorders, Fourth
Edition (DSM-IV). At 13 years of age, he had a complex partial seizure during which he lost consciousness and fell down. His interictal electroencephalogram showed sporadic spikes in the right temporal area (Figure 2a). He was diagnosed with localization-related epilepsy, and his attacks were well controlled with sodium valproate. The younger brother of the proband (IV-7) is 13 years old. At 10 years of age, his IQ was 52 according to the Wechsler Intelligence Scale for Children-Third Edition (WISC-III). At that time, he also showed complex partial seizure and his electroencephalogram showed right centrotemporal spikes (Figure $2 \mathrm{~b}$ ). He was treated with valproate and had no further seizures. The younger sister (IV-8 at 11 years of age) does not show any neurological symptoms. The younger sister (IV-9; 6 years of old) from a different father had difficulty with social interaction in kindergarten, showed restricted interests, and fulfilled the DSM-IV criteria for pervasive development disorder (PDD). The younger brother (IV-10) from the different father is 4 years old. He had a borderline developmental level according to the Kyoto Scale of Psychological Development and presented ADHD. The mother (III-4) of the proband was diagnosed with depression in her 20s but had no history of learning disorder.

For genetic analysis, blood samples were obtained from individuals III-4, IV-6, IV-7, IV-8, IV-9, and IV-10. The mother (III-4) gave 
written informed consent for the testing of her children (IV-6, IV-7, IV-8, IV-9, and IV-10) and herself. This research was approved by the bioethics committee for human gene analysis at the Jichi Medical University.

Array-comparative genomic hybridization (CGH) analysis was performed using Agilent Human Genome CGH 180K (Agilent Technologies, Inc, Santa Clara, CA, USA). A 584-kb duplication at Xp22.12 (nt. 19915 395-20 $498961 \mathrm{bp}$ ) was detected in the proband (IV-6). Subsequent analysis identified the same microduplication in the family members (III-4, IV-7, IV-8, IV-9, and IV-10). This indicated that this microduplication was inherited from the mother (III-4) (Figure 3b).

$\mathrm{X}$-chromosome inactivation patterns were examined using a methylation-specific polymerase chain reaction-based assay targeting the human X-linked androgen receptor gene (HUMARA) locus. ${ }^{5,6}$ $\mathrm{X}$-chromosome inactivation patterns were found to be random in the mother (41:59) and in the eldest sister (IV-8; 67:33). Non-informative results were obtained for the sister with PDD (IV-9) because of the homozygous HUMARA alleles. The mother had a 198 and a 207-bp HUMARA allele. The proband (IV-6) and his family members

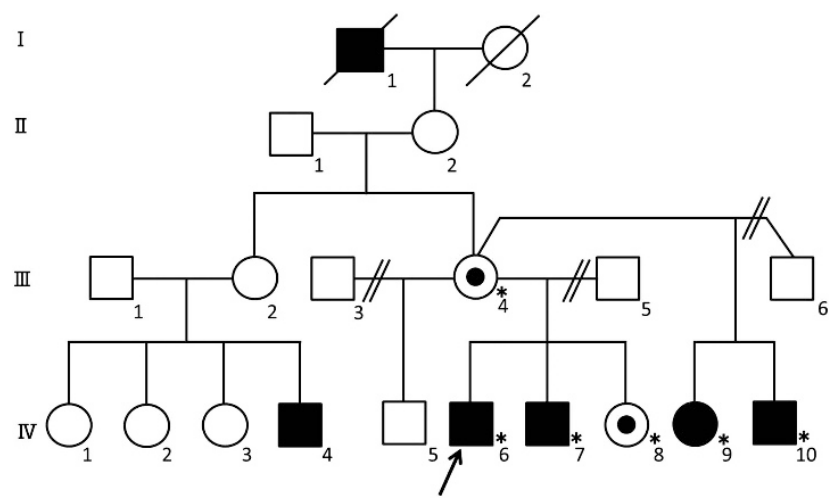

Figure 1 Pedigree of the family. Affected family members are indicated with black symbols; IV-6, IV-7, and IV-10 presented intellectual disability and IV-9 presented pervasive development disorder. Carrier females are indicated with a black dot inside their symbol. An arrow indicates the proband. An asterisk indicates individuals whose DNA were analyzed using array-comparative genomic hybridization (aCGH). Two lines drawn at a slant indicate a divorce.

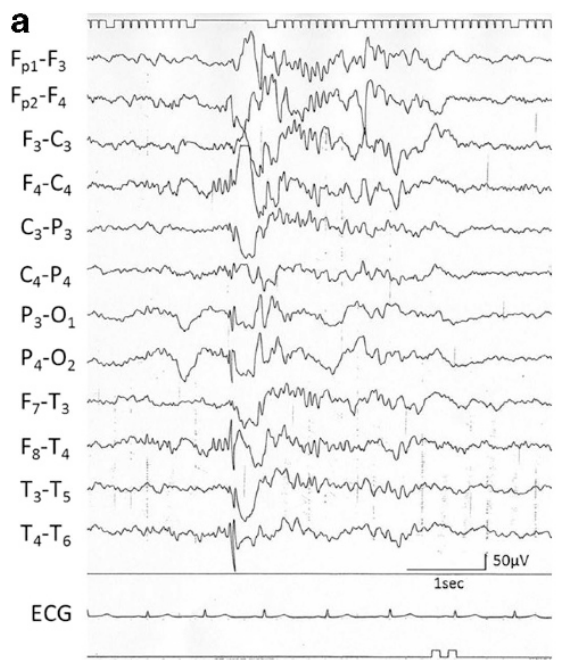

IV-7, IV-8, and IV-9 each had a 198-bp HUMARA allele, whereas IV10 had a 207-bp HUMARA allele (data not shown). These findings suggest that the 198-bp HUMARA allele was responsible for the 584$\mathrm{kb}$ duplication. Recombination occurs at a rate of about $10 \%$ in the $\mathrm{X}$-chromosome. ${ }^{7}$ It is assumed that the duplication observed in patient IV-10 was because of recombination between the HUMARA allele and duplicated areas in the oocyte.

Lymphoblasts from subjects III-4, IV-6, IV-7, IV-8, and IV-10 were analyzed using western blot; no sample was available for subject IV-9. The RPS6KA3 protein was detected with the mouse monoclonal E1 antibody (Santa Cruz Biotechnology, Inc, Dallas, TX, USA). Mouse polyclonal anti-tubulin antibody (Sony, Inc., Tokyo, Japan) was used as an internal control. Signal intensity was analyzed using the Image J software (http://rsbweb.nih.gov/ij/). Compared with the control samples, RPS6KA3 expression levels were 2.12-fold in subject IV-6, 1.77-fold in III-4, 1.91-fold in IV-7, 2.14-fold in IV-8, and 1.53-fold in IV-10 (Figure 3d).

The 584-kb microduplication identified in this study was confirmed to be inherited from the mother. The numbers of the genes involved in the Xp22.12 microduplication identified in this study were seven, and these are smaller than those of the previously reported microduplications (Figure 3c). ${ }^{3,4}$ These seven genes included uncharacterized LOC729609 (LOC729609), microRNA 23c (MIR23c), and small Cajal body-specific RNA 9-like (SCARNA9L), which are miscRNA genes, eukaryotic translation initiation factor $1 \mathrm{~A}$, X-linked (EIF1AX), chromosome X open reading frame 23 (CXorf23), and MAP7 domain containing 2 (MAP7D2), about which little is known; and RPS6KA3. RPS6KA3 is the only gene that may be responsible for neurological features of this family.

Concerning the symptoms, our cases and the two previously reported cases presented borderline IQ to mild ID. Madrigal et al. ${ }^{3}$ reported a male proband with mild ID and mild facial dysmorphism, who had an $800-k b$ duplication spanning from 19.82 to $20.65 \mathrm{Mb}$ at $\mathrm{Xq22.12}$. This duplication was also found in his asymptomatic mother, one sister, and two affected uncles. Tejada et al. ${ }^{4}$ identified a $1.05-\mathrm{Mb}$ duplication in a male proband with mild-to-borderline ID and ADHD. This duplication was also found in the proband's brother and two uncles, who also had mild-to-borderline ID. The RPS6KA3 mRNA and protein levels of the proband and the affected brother were twice as high as those of the father and carrier mother. In theory,

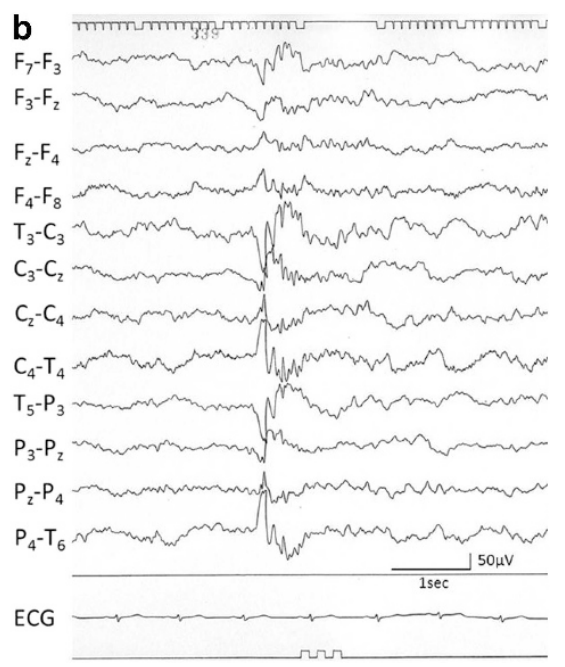

Figure 2 Electroencephalogram (EEG) of the patients. (a) EEG of the proband (IV-6) at 13 years of age, showing sporadic spikes in the right temporal area. (b) EEG of the brother (IV-7) at 11 years of age, showing right centrotemporal spikes. 


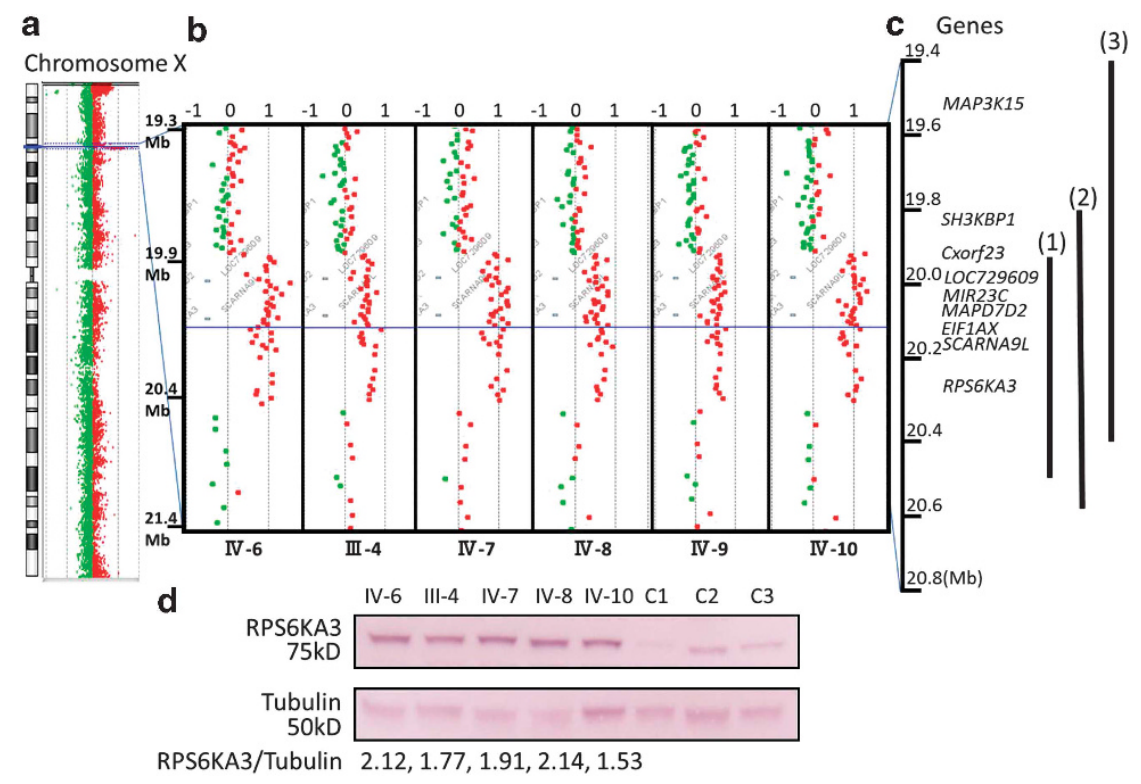

Figure 3 Array-comparative genomic hybridization (aCGH) results. (a) aCGH analysis of the entire X-chromosome in the proband (IV-6), showing a duplication on Xp22.12 (19.92-20.50 Mb). (b) Detailed views of the microarray plots for subjects IV-6, III-4, IV-7, IV-8, IV-9, and IV-10. The vertical axis shows megabases from the telomere of $\mathrm{Xp}$, and the horizontal axis shows the fold-change in copy number variation: 1 indicates duplication of one allele, and -1 indicates deletion of one allele. (c) The chromosomal position is indicated vertically as megabases from the telomere of Xp and existing genes. The bars-labeled (1), (2), and (3), respectively_indicate the region of duplication in our case, in the case reported by Madrigal et al., ${ }^{3}$ and in the case reported by Tejada et al. ${ }^{4}$ (d) Western blot analysis of RPS6KA3 (upper) and tubulin (lower) protein from lymphoblasts. The RPS6KA3 protein levels (bottom) were 2.12-fold in IV-6, 1.77-fold in III-4, 1.91-fold in IV-7, 2.14-fold in IV-8, and 1.53-fold in IV-10 compared with those in the controls (C1, C2, and C3).

the RPS6KA3 expression level is two times higher in affected males and 1.5 times higher in carrier females. The differences of RPS6KA3protein level between our cases and the cases described by Tajada et l. $^{4}$ might be because of altered methylation in lymphoblasts or limitations of the western blot analysis.

Similar to the proband reported by Tejada et al., ${ }^{4}$ in our present case, the proband (IV-6) and one brother (IV-10) presented ADHD. However, the presently described proband (IV-6) and his other brother (IV-7) also presented localization-related epilepsy. Epilepsy develops in $5 \%$ of CLS cases. ${ }^{1}$ In mice, mutation of one of the CREB (cAMP response element-binding protein)-response elements (CREBm) at endogenous Bdnf promoter IV resulted in reduced inhibitory synapse in the cortical neurons. ${ }^{8}$ Therefore, epilepsy is considered to be the phenotype of RPS6KA3 duplication. Concerning the female carriers, one female sibling (IV-9) presented PDD, and the mother (III-4) was diagnosed with depression. Psychiatric illness has been reported in $8.8 \%$ of the female CLS carriers. ${ }^{9}$ In previous cases with RPS6KA3 duplication, Madrigal et al. ${ }^{3}$ and Tejada et al. ${ }^{4}$ identified only normal female carriers with random X-chromosome inactivation patterns. Therefore, it is possible that PDD and depression are also symptoms of the RPS6KA3 duplication.

\section{CONFLICT OF INTEREST}

The authors declare no conflict of interest.

\section{ACKNOWLEDGEMENTS}

This work was supported by JSPS KAKENHI (grant numbers 22790996 and 23390275).

1 Pereira, P. M., Schneider, A., Pannetier, S., Heron, D. \& Hanauer, A. Coffin-Lowry syndrome. Eur. J. Hum. Genet. 18, 627-633 (2010).

2 Merienne, K., Jacquot, S., Pannetier, S., Zeniou, M., Bankier, A., Gecz, J. et al. A missense mutation in RPS6KA3 (RSK2) responsible for non-specific mental retardation. Nat. Genet. 22, 13-14 (1999).

3 Madrigal, I., Rodriguez-Revenga, L., Armengol, L., Gonzalez, E., Rodriguez, B., Badenas, C. et al. X-chromosome tiling path array detection of copy number variants in patients with chromosome X-linked mental retardation. BMC. Genomics 8, 443 (2007).

4 Tejada, M. I., Martinez-Bouzas, C., Garcia-Ribes, A., Larrucea, S., Acquadro, F., Cigudosa, J. C. et al. A child with mild X-linked intellectual disability and a microduplication at Xp22.12 including RPS6KA3. Pediatrics 128, e1029-e1033 (2011).

5 Kubota, T. A new assay for the analysis of X-chromosome inactivation in carriers with an X-linked disease. Brain. Dev. 23 (Suppl 1), S177-S181 (2001).

6 Kubota, T., Das, S., Christian, S. L., Baylin, S. B., Herman, J. G. \& Ledbetter, D. H. Methylation-specific PCR simplifies imprinting analysis. Nat. Genet. 16, 16-17 (1997).

7 Brooks, L. D. \& Marks, R. W. The organization of genetic variation for recombination in Drosophila melanogaster. Genetics 114, 525-547 (1986).

8 Hong, E. J., McCord, A. E. \& Greenberg, M. E. A biological function for the neuronal activity-dependent component of Bdnf transcription in the development of cortical inhibition. Neuron 60, 610-624 (2008).

9 Hunter, A. G. Coffin-Lowry syndrome: a 20-year follow-up and review of long-term outcomes. Am. J. Med. Genet. 111, 345-355 (2002). 